\title{
THE INTERACTION OF COMETARY PLASMA WITH INTERPLA- NETARY MEDIUM - A POST-HALLEY VIEW
}

\author{
Debi Prasad \\ Physical Research Laboratory \\ Navrangpura \\ Ahmedabad-380 009, India
}

\section{ABSTRACT}

The strong interaction of cometary plasma with the interplanetary medium results the disconnection events and other features observed in plasma tail and the coma of comets. The understanding of these features provide direct probe of the heliospheric magnetic field. However the interpretation of these events is not without ambiguity, as revealed in the recent work with comet Halley. In this paper a review of the "Post-Halley era" developments of the cometary plasma physics is presented.

\section{INTRODUCTION}

The study of cometary plasma have contributed significantly in understanding the nature of interplanetary medium (IPM). Based on the observation of plasma tail of comets in 1951 Biermann /1/ concluded the existance of continuous solar wind in the IPM at all solar latitudes. By far, the largest volume in the interplanetary space and largest span of time are covered by comets with plasma tails. Today, about more than 150 comets with plasma tails are knuwn, with the frequency of about one or even two new discoveries per year. In comparison with several methods, comets can be used to study a large volume of interplanetary medium $/ 2,3 /$. Therefore the understanding of the interaction of cometary plasma with the IPM is an essential part of the study of the basic plasma processes on the sun.

The comets are frazile coglomorates of various types of ices, made during the early stages of the solar system formation. On close approach to sun these bodies sublimate and strongly interact with solar wind producing the large coma and long plasma tails. The plasma tails consists of the ionic molecules such as $\mathrm{CO}^{+}, \mathrm{CO}_{2}$ and $\mathrm{H}_{2} \mathrm{O}^{+}$, appearing blue in color due to the characteristic emission 
(mostly of $\mathrm{CO}^{+}$and $\mathrm{CO}_{2}$ ). As a result of the complex interaction of solar wind with the cometary atmosphere, a number of features in the form of kinks, knots and condensations are formed in the tail and coma of the comets. The observations of various comets during last decade from the ground and from the space have enriched our knowledge in understanding many of these processes, with a single major understanding due to comet Halley in 1985-86.

\section{THE PLASMA BOUNDARIES}

The cometary plasma boundaries are excellently reviewed by Craveus 14/. A brief outline is given here. Figure 1 outlines various plasma boundaries. The solar extreme ultraviolet radiation ionizes the cometary neutrals, which slows down the solar wind due to the mass-loading. As a consequence the bow shock is generated with Mach number 2 (weak shock) at a distance of about $10^{6} \mathrm{~km}$ for Comet Halley (during the encounters) $/ 5 /$. The solar wind continues to be mass-loaded and to slow down, after being shocked and ulti $\overline{5}$ mately stagnates for cometocentric distances less than about $10^{5}$ $\mathrm{km}$ (for Halley's comet during encounters). Consequently the magnetic field strength increases, leading to the development of a magnetic barrier (or pile up region) in the vicinity of the stagnation region. Eventhough this process is described quite well by global, threedimensional MHD models, the measurements made by the instruments on various spacecrafts have shown that the structure of the stagnation region is rather complicated /6/. Hence presently this region is called cometopause. The imaging Fabry-Perot studies have resulted the line-of-sight velocity maps /7/ in $\mathrm{H} \alpha$ emission at $6563 \mathrm{~A}^{\circ}$, during an occassion when the $\mathrm{H}_{\alpha}$ intensity of this region was above the cometary continuum. These observations are valuable in understanding of the complex dynamics of cometopause. The theoretical studies involving the velocity map of this region is in progress 18/. As the cometocentric distance decreases the plasma becomes increasingly slow (the flow speeds $_{3}$ less than $20 \mathrm{~km} / \mathrm{s}$ ), dense (electron density about $10^{2}-10^{4} \mathrm{~cm}^{-3}$ between cometopause to 1000 $\mathrm{km}$ cometocentric distance) and cold (with electron temperature of $100 \mathrm{ev}$ at cometopause to $0.1 \mathrm{ev}$ at $1000 \mathrm{~km}$ cometocentric distance). This region is chemically complex and have magnetic field of about 60 at $10^{4} \mathrm{~km}$ due to declaration of the flow. In this region therefore, the collisional processes become important. The collisionpause is defined as the boundary within which the transport or dyhamical processes become less important than the collisional processes. The location of collisionopause for a given process is the region where the transport time $\tau$, equal the characteristic collision time $\boldsymbol{\tau}$. Therefore there exist ${ }^{\mathrm{T}}$ number of collisionpauses for different ionic species in the region between the cometppause and diamagnetic cavity, generally in the vicinity of $2 \times 10^{4} \mathrm{~km}$. In the innermost portion of the magnetic barrier, as a consequence of the balance between an outward ion-neutral frictional force on the plasma and an inward-directed magnetic pressure gradient force 
the diamagnetic cavity exists at a distance of about $4000 \mathrm{~km}$ from the nucleus (as observed by Giotto $4468 \mathrm{~km}$ in bound, $(4150 \mathrm{~km}$ out bound) $/ 9 /$. The comet tails are a consequence of mass loading processes which take place in the head of the comet and slows down the plasma flow in the sunward side, thus "hanging up" the field lines. The ICE spacecraft measured at about $8000 \mathrm{~km}$ downstream of comet Giacobini-Zinner, detecting the narrow layer of cold dense plasma in the middle of the tail and magnetic tail lobes, which were obviously due to drapped magnetic field lines $/ 10 /$.

Apart from these boundaries which are "Permanent" in nature, the boundaries intrinsic to solar wind carried into the cometary environment and some small scale features which are due to transient disturbances associated with plasma instabilities are also observed in comets. The later is poorly understood.

\section{MASS MOTION VERSUS WAVE MOTION}

Displacement of features in cometary plasma tails, obtained from a sequence of photographs, have been used to infer plasma motions with tailward velocities in the range of about 20 to $250 \mathrm{~km} / \mathrm{s} / 11$, $12 /$. However, from these observations it is not clear whether these apparent motions represent propagating disturbances such as magneto hydrodynamic waves moving down the tail or actual mass motions of the cometary ions or combination of both. This question can be resolved with Doppler measurements of ionic emissions. Huppler et al. /13/ have reported the doppler velocities of about 20-40 $\mathrm{km} / \mathrm{s}$ by measuring the line shift in $\mathrm{H}_{2} \mathrm{O}^{+}$emission in comet Kohoutek - thereby indicating the existance of mass motion. Debi Prasad et al. /7/ have observed with an imaging Fabry-Perot spectrometer, the motion of a plasma region with doppler velocities of about $30 \pm 10 \mathrm{~km} / \mathrm{s}$. The Fabry-Perot observations of Comet Halley in $\mathrm{H}_{2} \mathrm{O}^{+}$ emission by Scherb et al, /14/ have revealed the ionic accelerations about 37 to $300 \mathrm{~cm} \mathrm{sec}^{-2}$. From these measurements, it can be concluded that the tail particles are accelerated while moving in the plasma tail. However, it is still not clear whether the motion of the features represent the mass motion, since they show systematically lower acceleration in comparison with the Doppler measurements. In a future occasion it will be valuable to observe the Doppler velocities in the tail features. The ground based observations, specially with imaging Fabry-Perot spectrometers in $\mathrm{CO}^{+}$ emission lines will prove useful in these experiments.

\section{THE SHORT LIVED PLASMA FEATURES}

Historically the visual observations of the great comet of 1882 by Bernard /15/ can be regarded as the earliest record of the cometary transient features. During last two decades several such 
features have been identified $/ 7,16,18 /$ especially during the Comet Halley observations. Lynch and Russel /16/ have reported the observation of a blob like stucture at about $32000 \mathrm{~km}$ from the nucleus in the plane of sky, which did not show up in A'Hearn's /17/ measurements, thereby requiring a dissipation time scale of an hour. The image was obtained at fo $5800 \mathrm{~A}^{\circ}$ with a bandwidth of about $2000 \mathrm{~A}^{\circ}$. There is an evident of comet passing through the interplanetary sector boundary, prior to these observations. From three IUE low resolution spectra taken during 18-19 March 1986 Fedman et al. /18/ reported the sudden enhancement of $\mathrm{CO}^{+}$ intensity in the tailward side, at a distance of about $150000 \mathrm{~km}$ from the nucleus. The enhancement lasted only for 37 minutes. The calculations show that the photoionization and electron impact ionization processes cannot satisfactorily explain the event. It can be noted here that the comet crossed the interplanetary sector boundary on March 16.5 UT. With the imaging Fabry-Perot spectroscopic observations Debi Prasad et al. /7/ have recorded a short lived enhanced brightness region at position angle $160^{\circ}$ at a nucleocentric distance of about $2 \times 10 \mathrm{~km}$. The size of the region was $30,000 \mathrm{x}$ $20,000 \mathrm{~km}$ in the plane of sky. The brightness was prominent in $\mathrm{H}_{2} \mathrm{O}^{+}$emissions at $7000 \mathrm{~A}^{\circ}$. A Fabry-Perot interferogram is recorded in $\mathrm{H} \propto\left(6563 \mathrm{~A}^{\circ}\right)$ emission lines, following the $\mathrm{H}_{2} \mathrm{O}^{+}$imagines at the enhanced brightness region. Analyzing the interferogram, these authors have obtained the spatio-kinematic informations of this region. It was found that the radial recession velocity of the enhanced brightness region is $30 \pm 10 \mathrm{~km} / \mathrm{s}$ (cometocentric). The Doppler widths of the lines are 5 to $22 \mathrm{~km} / \mathrm{s}$. The relative differential velocity map is structured as shown in figure 2, with high \& low velocity regions clustered. Line splittings are seen in the sunward side. From the interferogram data, it is inferred that the dispersal time of the blob is about $30 \mathrm{~s}$, which is further supported by the contemporary observations. From the Sakigake and Giotto measurements and considering the motion of the comet, it is inferred that the southern part of the Haley's comet dipped first into the away sector in the changing polarity region. Therefore it is more likely that this event is a "tail disconnection type event" resulted due to the reconnection of field lines. Lutz and Wagner /19/ have reported that comet IRAS-Araki-Alcock 1983d showed an intense brightening event on 10 May 1983. The flare-like event manifested itself in increase of flux in continuum and emission band in 20 minutes during 0618 \& 0638 UT, preceded \& followed by periods of normal stability. The hypothesis of the ejection of rock-like particles and the encounter of interplanetary shock as the precursor of this event are unsatisfactory. Since the sun was quite the solar cause can be rulled out. It is proposed that venus like interaction of interplanetary magnetic field with the coma can cause such events $122 /$. In absence of any observational evidence for supporting this mechanism, the reconnection mechanism, while the comet passes through the interplanetary magnetic field can also be considered. 
Reviewing the well documented transient plasma events therefore indicate that the magnetic field reconnection process must be responsible for most if not all of the events. However, detailed theoretical studies of these events are yet to be done.

\section{ACKNOWLEDGEMENTS}

The author would like to thank Drs.J.N.Desai and T.Chandrasekhar for their critical comments.

\section{REFERENCES}

1. Biermann, L., 1951, Z.Astrophys., 29, 274.

2. Debi Prasad C., 1988, Ph.D thesis, Guj.University, India.

3. Hoeksema, J.C., 1989, Adv.Space Res., 9, (4) 141.

4. Cravens, T.E., 1989, Adv.Space Res., 9, (3) 293.

5. Anderson, K.A., Carlson, C.W., Curtis, D.W., Lin, R.P., Re'me, H., Sanvaud, J.A., d'Uston, C., Korth A., Richter, A.K., and Mendis, D.A., 1987, Astron. Astrophys., 187, 290.

6. Re'me, H., Sanvaud, J.A., d'Uston, C., Cros.A., Anderson, K.A., Carlson, C.W., Curtis, D.W., Lin, R.P., Korth A., Richter, A.K., and Mendis, D.A., 1987, Astron. Astrophys., $187,33$.

7. Debi Prasad C., Chandrasekhar T., Desai, J.N., Ashok N.M., 1989, J. Astron. Astrophys., 10, 1.

8. Khara A., Personal communication.

9. Ip.W.H., and Axford, W.I., 1987, Nature, 325, 418.

10. Meyer-Vermet, N., Conturier, P., Hoang, S., Perche, C., Steinberg, J.L., Fainberg, J. and Meetre, C., 1986, Science, 232,370 .

11. Jockers, K., 1985, Astron.Astrophys., Suppl. Ser., 62, 791.

12. Celnik, W.E. and Th.Schmidt-Kaler, 1987, Astron. Astrophys., $187,223$.

13. Huppler, D., Reynolds, R.J., Roesler, F.L., Scherb, F., Trauger, J., 1975, Ap.J., 202, 276.

14. Scherb, F., Magee-Sauer, K., Roesler, F.L. and Harlander J., 1989, Icarus, submitted.

15. Bernard, E.E., 1893, Astron. Assoc., J., 9, 59-63.

16. Lynch and Russell, 1988, Pub. Astro. Soc. Pac., 100, 1122.

17. A'Hearn, M.F., Personal Communication.

18. Feldmann et al., 1986, Nature, 324, 646.

19. Lutz, B.L., Wagner, R.M., 1986, Ap. J., 308, 993.

20. Russel, C.T., Luhmann, J.G., Baker, D.N., 1987, Geophys. Res., Lett., 14, 991 . 


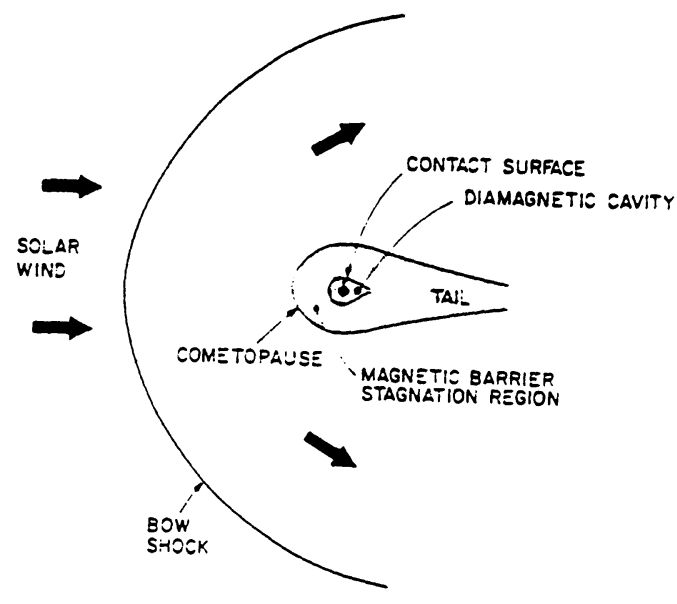

Fig. 1 : Schematic of plasma boundaries of an active comet.

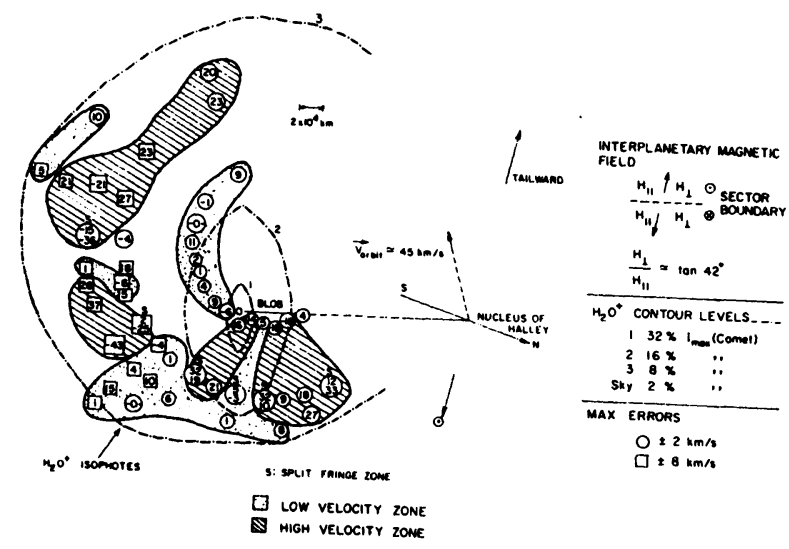

Fig. 2 : Doppler shift dispersion ${ }_{+}$velocity distribution in the $\mathrm{H}_{2} \mathrm{O}^{+}$blob region. $\mathrm{H}_{2} \mathrm{O}^{+}$isophotes contour levels are drawn from the cometary image taken earlier to the interferogram. 